\title{
Assessment of CFD Hypersonic Turbulent Heating Rates for Space Shuttle Orbiter
}

\author{
William A. Wood* \\ NASA Langley Research Center, Hampton, Virginia, 23681 \\ A. Brandon Oliver ${ }^{\dagger}$ \\ NASA Johnson Space Center, Houston, Texas, 77058
}

\begin{abstract}
Turbulent CFD codes are assessed for the prediction of convective heat transfer rates at turbulent, hypersonic conditions. Algebraic turbulence models are used within the DPLR and LAURA CFD codes. The benchmark heat transfer rates are derived from thermocouple measurements of the Space Shuttle orbiter Discovery windward tiles during the STS119 and STS-128 entries. The thermocouples were located underneath the reaction-cured glass coating on the thermal protection tiles. Boundary layer transition flight experiments conducted during both of those entries promoted turbulent flow at unusually high Mach numbers, with the present analysis considering Mach 10-15. Similar prior comparisons of CFD predictions directly to the flight temperature measurements were unsatisfactory, showing diverging trends between prediction and measurement for Mach numbers greater than 11. In the prior work, surface temperatures and convective heat transfer rates had been assumed to be in radiative equilibrium. The present work employs a one-dimensional time-accurate conduction analysis to relate measured temperatures to surface heat transfer rates, removing heat soak lag from the flight data, in order to better assess the predictive accuracy of the numerical models. The turbulent CFD shows good agreement for turbulent fuselage flow up to Mach 13. But on the wing in the wake of the boundary layer trip, the inclusion of tile conduction effects does not explain the prior observed discrepancy in trends between simulation and experiment; the flight heat transfer measurements are roughly constant over Mach 11-15, versus an increasing trend with Mach number from the CFD.
\end{abstract}

\section{Nomenclature}

Symbols

$h \quad$ Altitude, $\mathrm{km}$

$q \quad$ Heat transfer rate, $\mathrm{W} / \mathrm{cm}^{2}$

$T \quad$ Temperature, $\mathrm{K}$

$\alpha \quad$ Angle of attack, degrees

$\beta \quad$ Sideslip angle, degrees

\section{Acronyms}

CFD

DPLR

FRCI-12

HYTHIRM

LAURA

LI-900

\author{
Computational fluid dynamics \\ Data-Parallel Line Relaxation CFD code \\ Fibrous refractory composite insulation tiles \\ Hypersonic Thermodynamic Infrared Measurements \\ Langley Aerothermodynamic Upwind Relaxation Algorithm CFD code \\ Tile material developed by Lockheed Missiles and Space Company
}

\footnotetext{
*Aerothermodynamics Branch, AIAA senior member.

${ }^{\dagger}$ Applied Aeroscience and CFD Branch.
} 


$\begin{array}{ll}\text { OML } & \text { Outer mold line } \\ \text { RCC } & \text { Reinforced carbon-carbon } \\ \text { RCG } & \text { Reaction-cured glass } \\ \text { RTV } & \text { Room-temperature vulcanizing adhesive } \\ \text { STS } & \text { Space Transportation System } \\ \text { TC } & \text { Thermocouple }\end{array}$

\section{Introduction}

Boundary layer transition experiments were conducted on the Space Shuttle orbiter Discovery during the STS-119 and 128 reentries. ${ }^{1}$ The experiments included a boundary layer trip that was 0.25 inches tall on STS-119 and 0.35 inches tall on STS-128. The trips produced boundary layer transition at nominally Mach 15 and Mach 18 on STS-119 and STS-128, respectively. The on-board instrumentation package consisted of ten thermocouples mounted in the windward thermal protection tiles of the port wing. Discovery has an additional six thermocouples in the windward tiles as part of the standard vehicle instrumentation package. More recent flights of the boundary layer transition experiment were conducted on STS-131, 133, and $134{ }^{2}$ but are not considered herein.

In conjunction with the flight experiments, during STS-119 and 128 global images of the windward temperatures were taken using infrared cameras on remote platforms by the Hypersonic Thermodynamic Infrared Measurements (HYTHIRM) project. ${ }^{3}$ The HYTHIRM temperature measurements are of the orbiter vehicle outer skin, whereas the thermocouple measurements are under the reaction-cured glass coating of the thermal protection tiles. HYTHIRM has also acquired images of orbiter reentries during the STS-125, 131, 132, 133, and 134 missions.

Related to the Space Shuttle orbiter boundary layer transition experiments a special session is being held at the 42nd AIAA Thermophysics Conference, June 2011, entitled, "Entry Aerothermodynamics: Recent Flight Measurements." Coverage of the boundary layer transition experiment includes the flight data ${ }^{2}$ associated wind tunnel tests, ${ }^{4}$ and the present comparisons with numerical simulations. HYTHIRM topics include a project perspective, ${ }^{5}$ the conversion of radiant intensities into temperature measurements, ${ }^{6}$ and mission operations. ${ }^{7}$

The present report assesses computational fluid dynamics codes for the simulation of hypersonic turbulent flows, using convective heating rates as the metric. This assessment against flight data represents a contribution toward the validation of hypersonic turbulence modeling for entry simulations. With regard to HYTHIRM, improved understanding of the CFD accuracy is helpful both pre-flight, for the prediction of the expected range of radiance intensities from the vehicle heatshield, and post-flight, for assessment of the inferred surface temperature measurements. Additionally, the present thermal analysis of the vehicle tiles provides a quantification of the temperature differences measured on the vehicle surface by HYTHIRM and by the thermocouples, recessed within the surface.

Prior assessments of CFD against the STS- $119^{8}$ and $128^{9}$ thermocouple temperature measurements proved to be inconclusive for Mach numbers greater than 11. Although the measured and predicted temperatures were generally within $5 \%$ for Mach numbers less than 11, the predicted temperature trends diverged from the measurements for Mach numbers greater than 11.

For example, figure 1 is taken from the data of reference 8, showing CFD predictions compared to a thermocouple history from STS-119. The symbols represent various CFD simulations, and the line is the thermocouple temperature history. For the reentry flight path, the Mach number axis is traversed from high to low. It is notable that the flight data changes trend at Mach 11 but the CFD predicted temperature trend does not.

Figure 2 is another comparison of turbulent CFD predictions with a thermocouple temperature history, this time with STS-128 flight data. ${ }^{9}$ Again there is a difference in trend between turbulent CFD and measurement for Mach numbers greater than 11.

Some possible reasons for why the turbulent simulations do not follow the measured temperature trends at the higher Mach numbers are inadequate transitional boundary layer modeling, improper transition onset location specification, neglect of the tile transient thermal response, and non-continuum effects on either the fluid-solid interface or the nature of turbulent flow in general. The recasting of the comparisons from temperature to convective heat transfer, by way of transient thermal analyses of the tiles that contain the thermocouples, is intended to address the issue of thermal lag potentially invalidating the assumption of a 


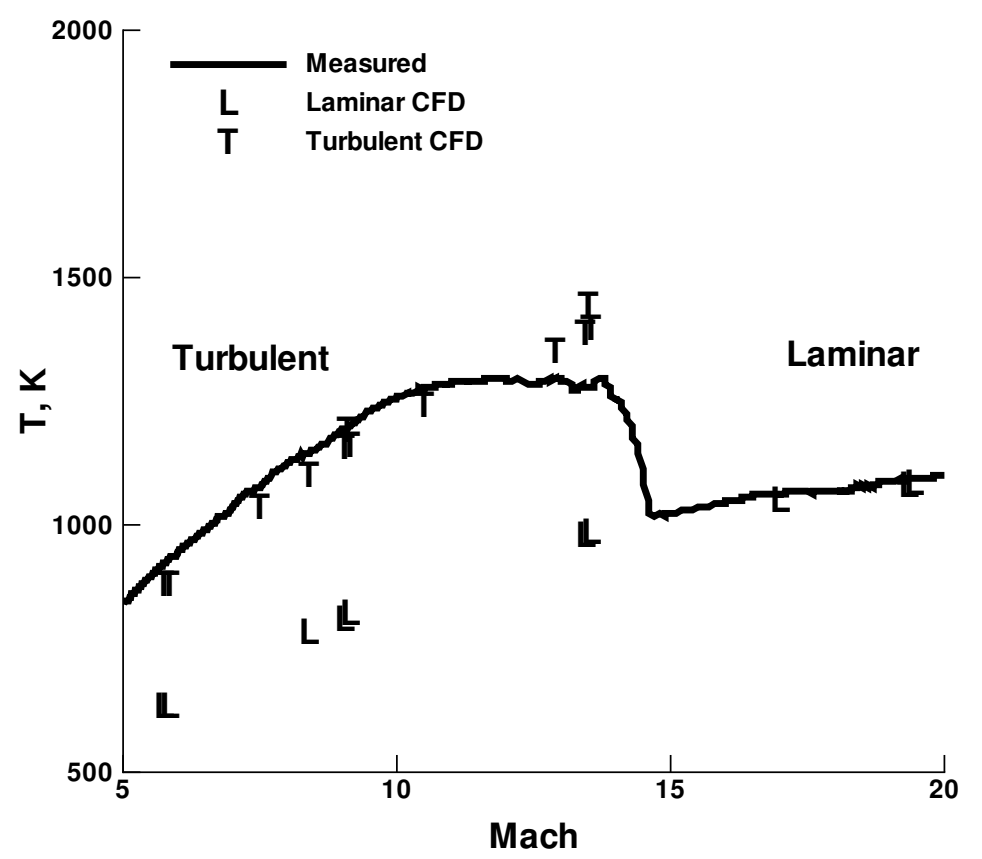

Figure 1. STS-119 sample temperature versus Mach number comparison between CFD predictions (symbols) and thermocouple measurements (data line), from reference 8.

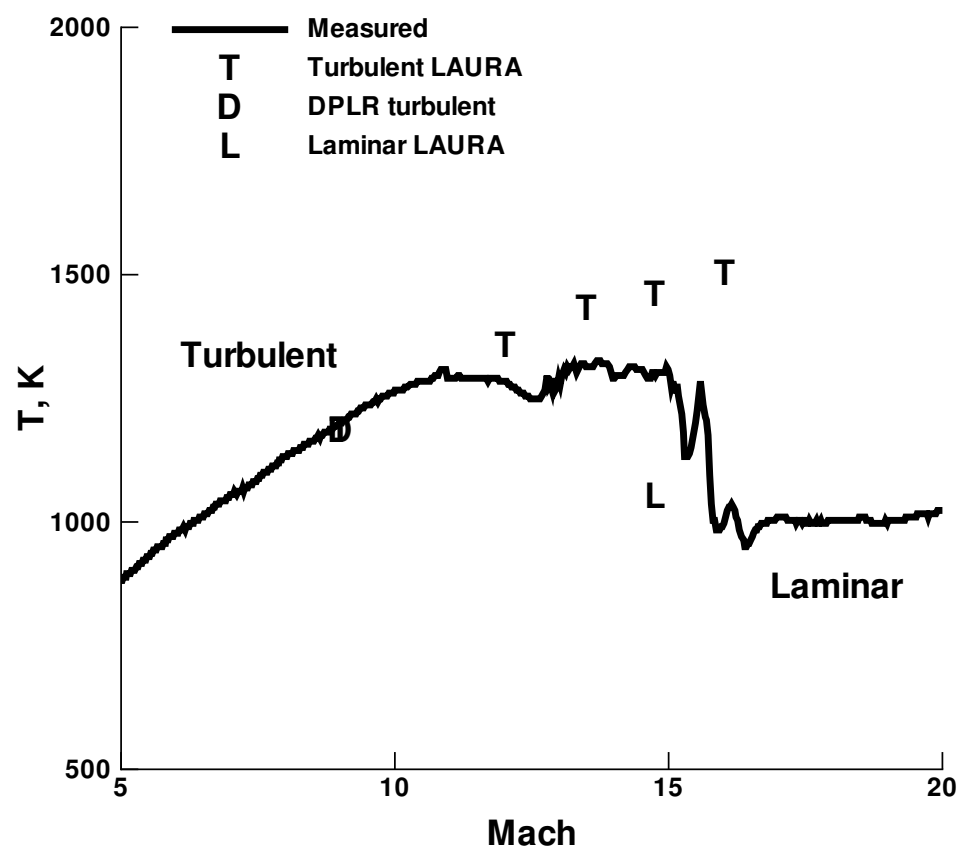

Figure 2. STS-128 sample temperature versus Mach number comparison between CFD predictions (symbols) and thermocouple measurements (data line), from reference 9. 


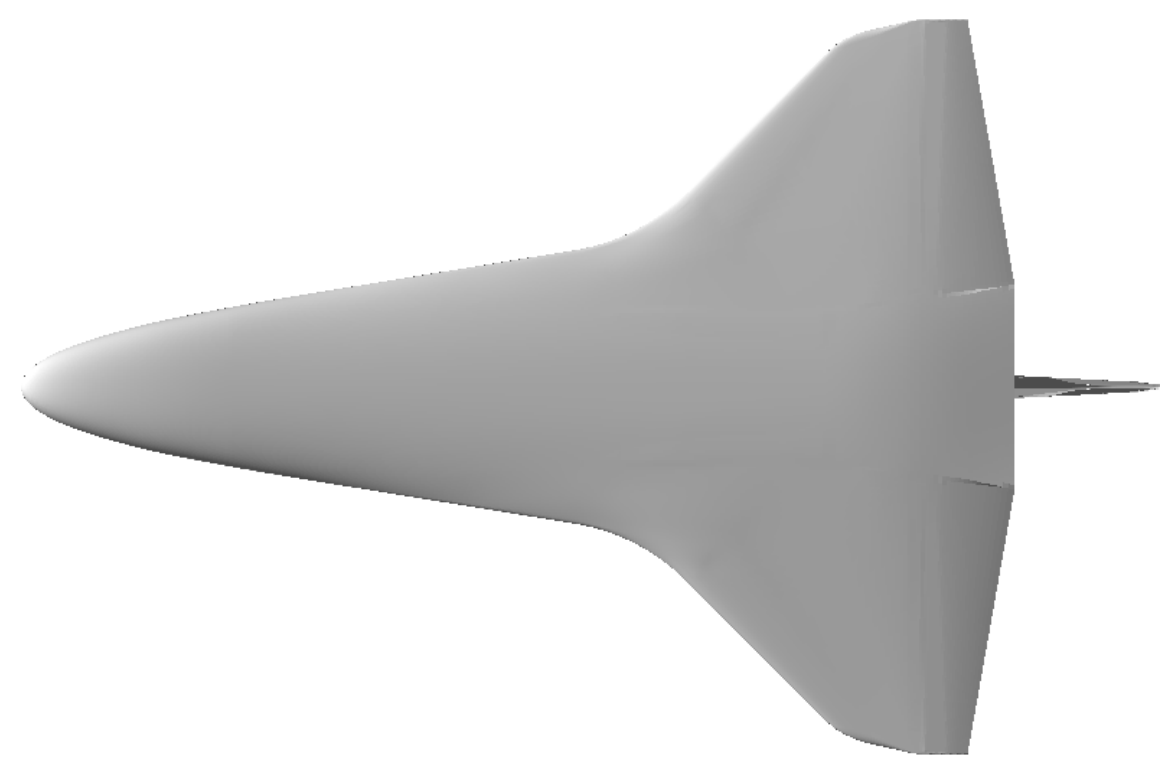

Figure 3. Windward planform of CFD vehicle geometry. Primary omission is the body flap.

radiative equilibrium surface energy balance from the previous studies.

\section{CFD simulations}

The numerical simulations are from the Data Parallel Line-Relaxation (DPLR) software ${ }^{10}$ and the Langley Aerothermodynamic Upwind Relaxation Algorithm (LAURA). ${ }^{11,12}$ Details of the simulations, including verification, have been presented in references 8 and 9. The database of CFD flow field simulations used in the present report were originally generated during the studies reported in references 8 and 9 . The results from DPLR and LAURA matched very closely, and are grouped together as "CFD" results in the present report.

Both DPLR and LAURA are finite-volume upwind-flux schemes for solving the Navier-Stokes ${ }^{13,14}$ equations with non-equilibrium chemistry - a five-species air model in thermal equilibrium for the present application. The entire vehicle was modeled with reaction-cured glass (RCG) surface properties; RCG is the exterior coating on the windward, black tiles of the orbiter. The simulations were steady state for discrete trajectory points, with the surface temperatures determined from a surface energy balance between the convective heat transfer and re-radiation at an emissivity of $85 \%$ plus conduction into the tiles at a rate of $4.5 \%$ of the convective rate. The CFD surface temperature boundary condition did not incorporate the flight thermocouple readings as any type of Dirichlet condition.

The simulations have either fully-laminar or fully-turbulent boundary layers. Turbulent simulations are fully-turbulent from the nose, wet the entire vehicle, and use the steady-state Reynolds-averaged NavierStokes equations. With LAURA, the algebraic Cebeci-Smith ${ }^{15}$ turbulence model was employed. With DPLR, the algebraic Baldwin-Lomax ${ }^{16}$ turbulence model was employed.

For laminar simulations, the CFD codes, the solution processes, the verification techniques, and the validation data have been accepted for use during Space Shuttle missions by the Orbiter Configuration Control Board. ${ }^{17}$ Previous laminar Orbiter solutions of a similar nature and application, using the same CFD codes, have been reported ${ }^{18}$ in the context of the Columbia accident investigation.

The computational volume grids contain about 12 million cells, and are solution-adapted for bow-shock alignment and boundary-layer clustering. Simplifications were made to the aft end of the vehicle, resulting in the windward plan-form shown in figure 3. The elevons are undeflected, the elevon gaps are not modeled, the body flap is omitted, and the trailing edge of the elevons has been extended to meet the body-flap truncation. The main engine and orbital maneuvering nozzles are omitted. The flow-field domain encompasses the bow shock and ends at the wing trailing edge. The OML is smooth, in that geometry perturbations smaller than a few inches are quilted over. In particular, the boundary layer trip on the wing is not discretely modeled 
Table 1. STS-119 trajectory points. Flight sideslip angles are listed, but all CFD simulations set $\beta=0$.

\begin{tabular}{rccc} 
Mach & $h, \mathrm{~km}$ & $\alpha,{ }^{\circ}$ & $\beta,^{\circ}$ \\
\hline 19.4 & 66.0 & 39.2 & 0.1 \\
17.0 & 62.6 & 42.1 & 0.5 \\
13.5 & 56.2 & 39.0 & 0.0 \\
12.9 & 55.5 & 39.3 & 0.1 \\
10.5 & 52.3 & 39.2 & 0.2 \\
9.1 & 49.9 & 38.3 & 0.2 \\
7.5 & 46.7 & 35.2 & 0.0 \\
5.8 & 40.2 & 28.0 & 0.0
\end{tabular}

Table 2. STS-128 trajectory points. Flight sideslip angles are listed, but all CFD simulations set $\beta=0$.

\begin{tabular}{rrrr} 
Mach & $h, \mathrm{~km}$ & $\alpha,{ }^{\circ}$ & \multicolumn{1}{c}{$\beta,^{\circ}$} \\
\hline 16.0 & 59.8 & 40.2 & 0.1 \\
14.8 & 58.2 & 42.0 & 0.0 \\
13.5 & 55.9 & 41.0 & -0.3 \\
12.0 & 54.2 & 40.1 & -0.4 \\
9.0 & 49.0 & 38.3 & -0.3
\end{tabular}

in the CFD geometry; see Tang ${ }^{19}$ for CFD modeling of the boundary layer trip and vicinity flow field.

The CFD simulations of STS-119 were originally performed for reference 8, with the eight trajectory points listed in table 1 . The CFD simulations of STS-128 were originally performed for reference 9 , at the five trajectory points listed in table 2 .

\section{Flight data}

Details of the boundary layer transition flight experiments and the thermocouple measurements can be found in references 1 and 2 . The experiments consisted of a discrete roughness element installed on the port wing of Discovery, 0.25 inches in height for STS-119 and 0.35 inches in height for STS-128. The boundary layer trips were designed to cause transition onset in the Mach 15-18 range, and appeared to have functioned as designed. ${ }^{2}$ The flight instrumentation package consisted of a total of sixteen thermocouples embedded in the windward thermal protection tiles. Ten of these thermocouples were added specifically for the flight experiment, placed in conjunction with the boundary layer trip on the port wing.

The present analysis selects a subset of the thermocouple data, chosen for the indication of high Mach number transition. From STS-119, TC-1, 2, and 90 were chosen. From STS-128, TC-2, 6, 80, and 90 were chosen. Additionally, TC-97 from STS-128 is shown for context with TC-80; TC-80 and 97 are located symmetrically about the vehicle centerline. Boundary layer transition is inferred from thermocouple measurements as a sharp rise above the expected laminar level. Details of the thermocouple installations are in table 3. The two tile material types, FRCI-12 and LI-900, are particular formulations of the hightemperature reusable surface insulation tiles that are used as part of the orbiter's thermal protection system. Where indicated, the thermocouple installations at the tile-to-RCG interface have been verified by x-ray with the RCG thickness measured. TC-80, 90, and 97 are assumed to have a $760 \mu \mathrm{m}$ RCG thickness. The locations of the thermocouples on the windward side of Discovery during STS-128 are shown in figure 4. The thermocouple numbers shown in black of figure 4 were used in the present work, and were located in the same positions during STS-119. The thermocouple numbers in blue were not used in the present work, and two of them, TC-3a and 8a, were located in slightly different positions on STS-119.

An example of the flight-measured temperatures for TC-2, 6, 80, and 90 from the STS-128 reentry are shown in figure 5. The flight trajectory traverses the Mach number domain in figure 5 from high to low. 
Table 3. Thermocouple names and locations, with material thicknesses.

Full name Short name RCG, mm Tile, mm Material Note

\begin{tabular}{lccccl}
\hline V07T9751A & TC-1 & 0.91 & 50 & LI-900 & Downstream of trip, verified by x-ray. \\
V07T9752A & TC-2 & 0.86 & 49 & LI-900 & Downstream of trip, verified by x-ray. \\
V07T9756A & TC-6 & 0.61 & 46 & LI-900 & Downstream of trip, verified by x-ray. \\
V07T9480A & TC-80 & 0.76 & 28 & FRCI-12 & Port mid-fuselage. \\
V07T9590A & TC-90 & 0.76 & 27 & LI-900 & Aft centerline. \\
V07T9597A & TC-97 & 0.76 & 28 & FRCI-12 & Starboard mid-fuselage.
\end{tabular}

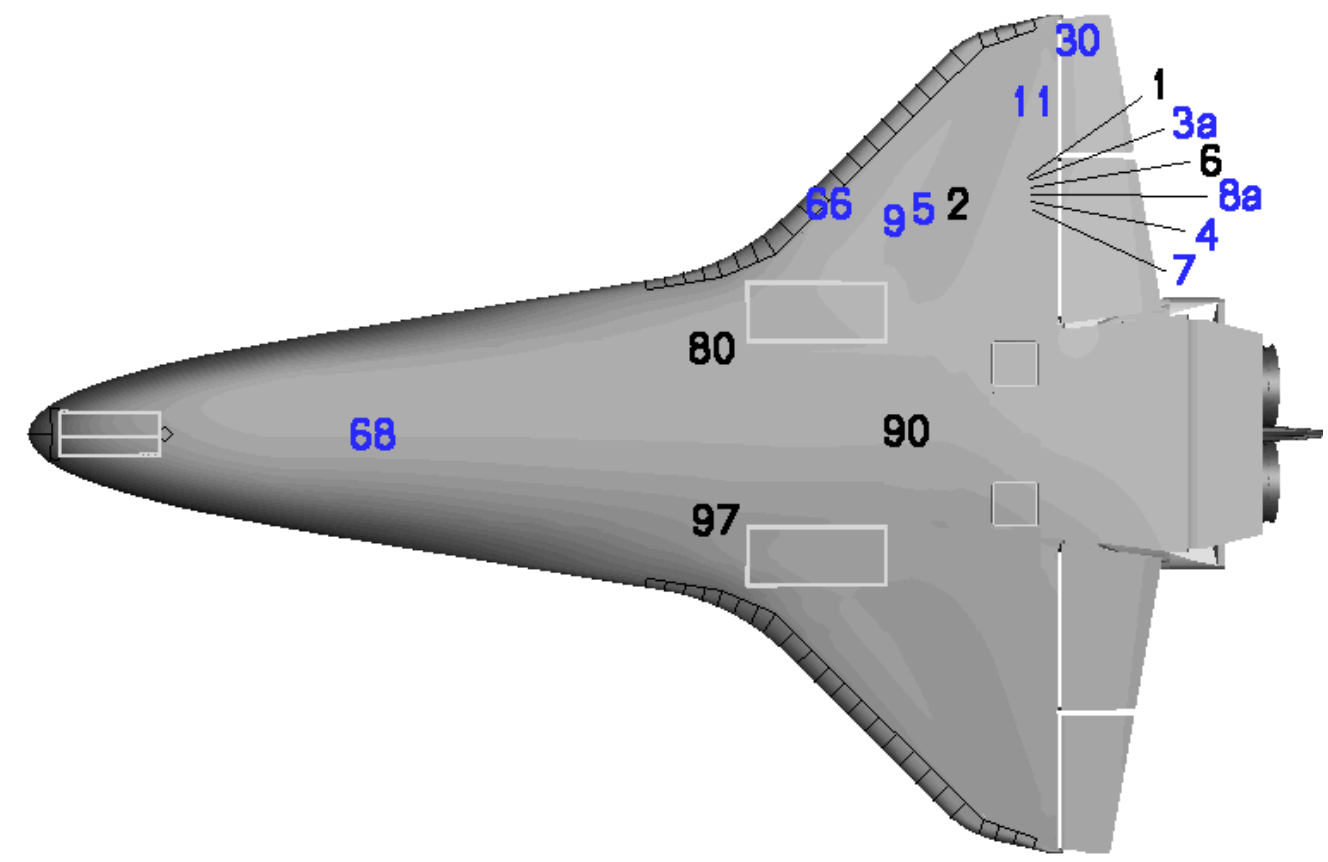

Figure 4. STS-128 thermocouple layout on Discovery, windward view. TC-9 is at the location of the flight experiment boundary layer trip. The black, not blue, thermocouples were used in the present analyses.

The temperature trends at Mach 20 are indicative of laminar flow, whereas the trends at Mach 5 indicate turbulent flow. Between Mach 13-16, the sharp rises in temperature, reading the Mach axis from high to low, indicates boundary layer transition onset. The start of fully-developed turbulent flow is not so easily identified; recall that the present objective is to assess numerical simulations of fully-developed turbulent flow.

\section{Conduction modeling}

Heat conduction through the thermal protection tiles was simulated using the Charring Ablating Thermal Protection Implicit System Solver. ${ }^{20}$ This code solves the charring ablator, general porous flow, and heat transfer problems in serial or parallel. In the present application, the tiles neither char nor ablate. The code uses the first and second order implicit time integrators and the Galerkin FEM spatial discretization on unstructured hybrid meshes. The governing equations are fully coupled and solved implicitly with Newton's method (with exact Jacobian terms), and the Generalized Minimal Residual method (GMRES) is used to solve the linear system. The exact Jacobian terms are calculated through the use of the complex-step (or complex perturbation) method rather than being analytically derived. This enables optimal convergence of the nonlinear solver. An inverse heat conduction capability is included which permits estimation of surface heating given in-depth temperature measurements using a sequential function specification algorithm. ${ }^{21}$ 


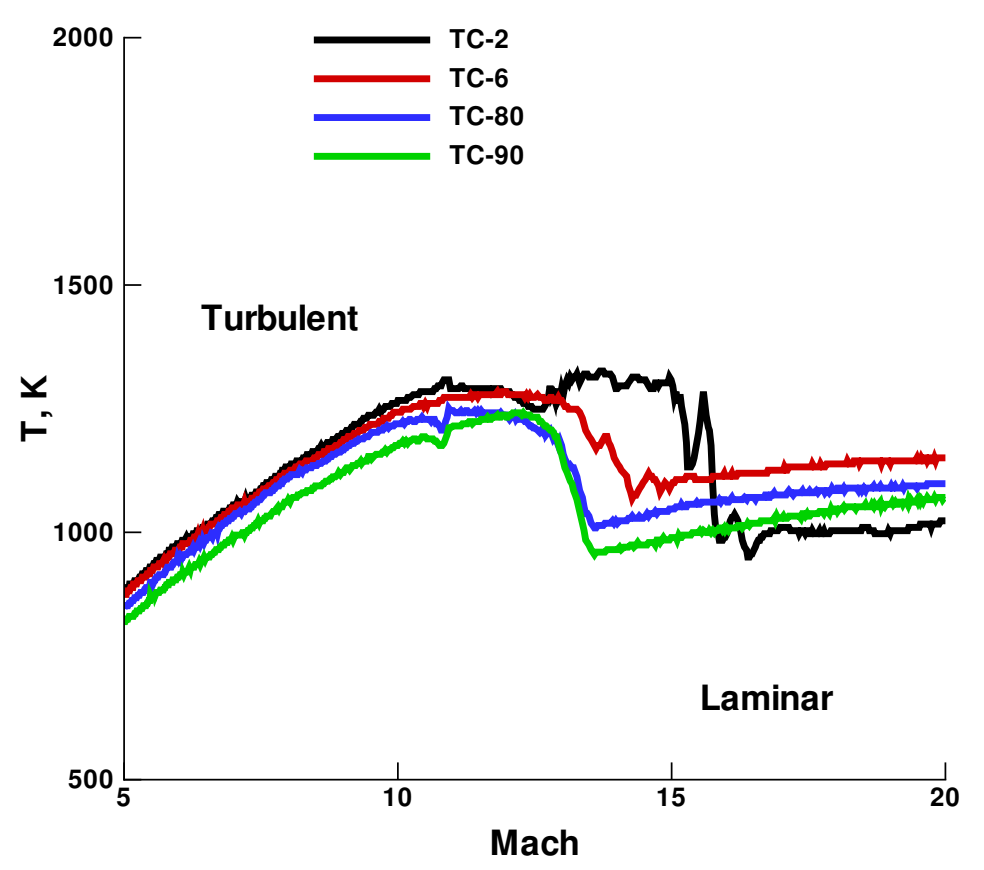

Figure 5. STS-128 thermocouple temperature histories for TC-2, 6, 80, and 90.

The code has been through a rigorous verification process to verify correct implementation of the governing equations.

The present one-dimensional thermal analyses were conducted on a stack-up consisting of an RCG coating, nominal tile material, a densified tile layer, a room-temperature vulcanizing (RTV) adhesive bond, the strain isolation pad, an inner RTV bond, and an aluminum skin. This stack-up is notionally shown in figure 6, which is not drawn to scale. For the FRCI-12 tiles, the numerical grid had 10 cells in the RCG coating, 300 cells in the nominal tile material, 25 cells in the densified tile layer, 5 cells in the outer RTV bond, 25 cells in the strain isolation pad, 5 cells in the inner RTV bond, and 20 cells in the aluminum skin. Results for the LI-900 tiles were found to be insensitive to the tile backing material, and so the numerical grid was simplified to 10 cells in the RCG coating with 180 cells in the nominal tile material, without distinguishing a densified layer. Pressure-dependent tile material properties were used and the trajectory marching time step was one second.

An adiabatic condition was applied to the back face and a re-radiation condition to a $300 \mathrm{~K}$ sink was applied to the front face. For the direct method, the thermocouple temperatures were applied on the front face. For the inverse method, a specified flux was applied and modified at each time step by the inverse algorithm to minimize the error between the thermocouple data and the temperature in the thermal model at the RCG-to-tile junction over a five second period. The convective heat flux is taken to be the balance of the conduction flux into the material and the re-radiation flux away from the surface. The solid was initialized to the thermocouple temperature from the time of entry interface during the trajectory.

The raw thermocouple traces, figure 5, required filtering to obtain smooth heat flux values. A $4^{\text {th }}$ order Savitzky-Golay ${ }^{22,23}$ linear filter with $0^{\text {th }}$ order smoothed derivatives was used on a window of 51 temporal points spaced at one second intervals. Due to the least-squares minimization nature of the inverse algorithm, some additional filtering was built in and results in smoother heat flux results.

From examination of the CFD surface pressure predictions, the surface pressure coefficient is roughly uniform over the present region of interest on the orbiter windward side, and relatively constant through the Mach number range of the trajectory being considered here, as the angle of attack remains nominally constant. The surface pressure for all conduction cases was therefore obtained using a 0.9 coefficient of pressure throughout the trajectory. 


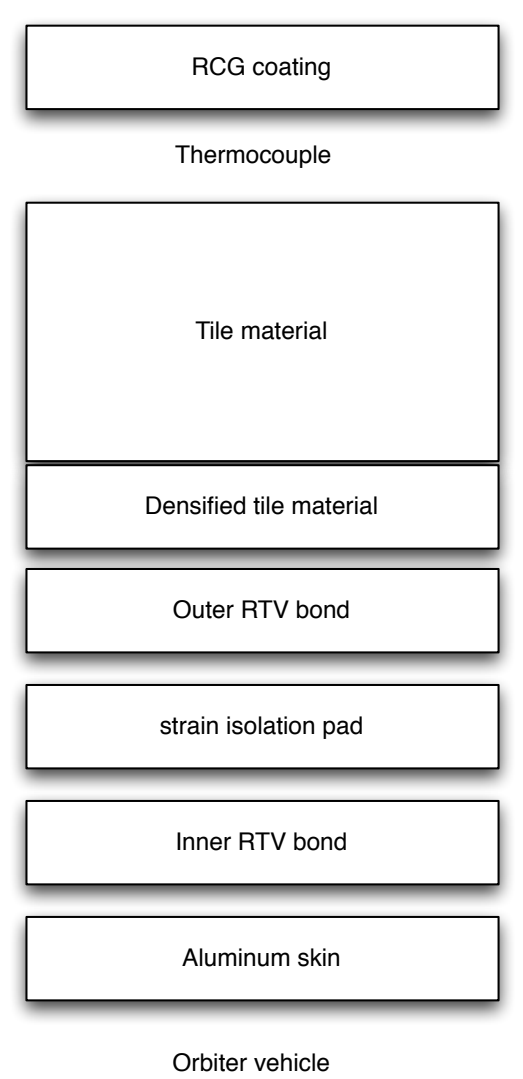

Figure 6. Stack-up of tile installation used for conduction modeling. Material thicknesses are not to scale.

\section{Results}

The focus of the present work is to assess the predictive ability of turbulent CFD simulations for convective heat transfer in the Mach 10-15 range. Because the flight data is from reentry trajectories, the Mach number is not the only free stream parameter changing; as the Mach number decrease, so does the velocity, while the Reynolds number, density, and dynamic pressure are increasing. The following figures span Mach 520 to provide context outside the range of focus. Laminar CFD predictions are also included in the plots for context. The present assessment sheds light on the adequacy of the present modeling for predictions within this flight regime. The numerical models are of fully-developed turbulence, without a transitional flow region; the true flow has a transition running length. The computations wet the entire vehicle with turbulent flow, but the tripped flow during flight forms a wedge of turbulence within an otherwise laminar flow. The thermocouple measurements clearly show when non-laminar conditions begin, but thermocouples of the type here are insufficient to clearly define a start to fully-developed turbulent flow.

The plots can be read in a reentry flight trajectory sense by traversing from high (right) to low (left) Mach number. The data line is the surface convective heat transfer as computed from the thermocouple readings. The symbols represent corresponding CFD predictions, $T$ for turbulent simulations and $L$ for laminar simulations. Where multiple CFD models were applied at the same trajectory point, the plotting symbols have been slightly jittered as described in reference 8 to make the symbol overlap visually distinguishable.

Convective heat transfer rates are plotted versus Mach number for TC-1 during the STS-119 reentry in figure 7. TC-1 is located downstream of the boundary layer trip, almost to the elevons. That positioning ensured that TC-1 would experience the earliest transition. Boundary layer transition begins at about Mach 16, with a rapid rise in heating to above $15 \mathrm{~W} / \mathrm{cm}^{2}$. The heating remains relatively constant over the Mach 11-15 range, before dropping along a traditional turbulent heating trend for the orbiter trajectory ${ }^{24}$ from Mach 11 downward. 


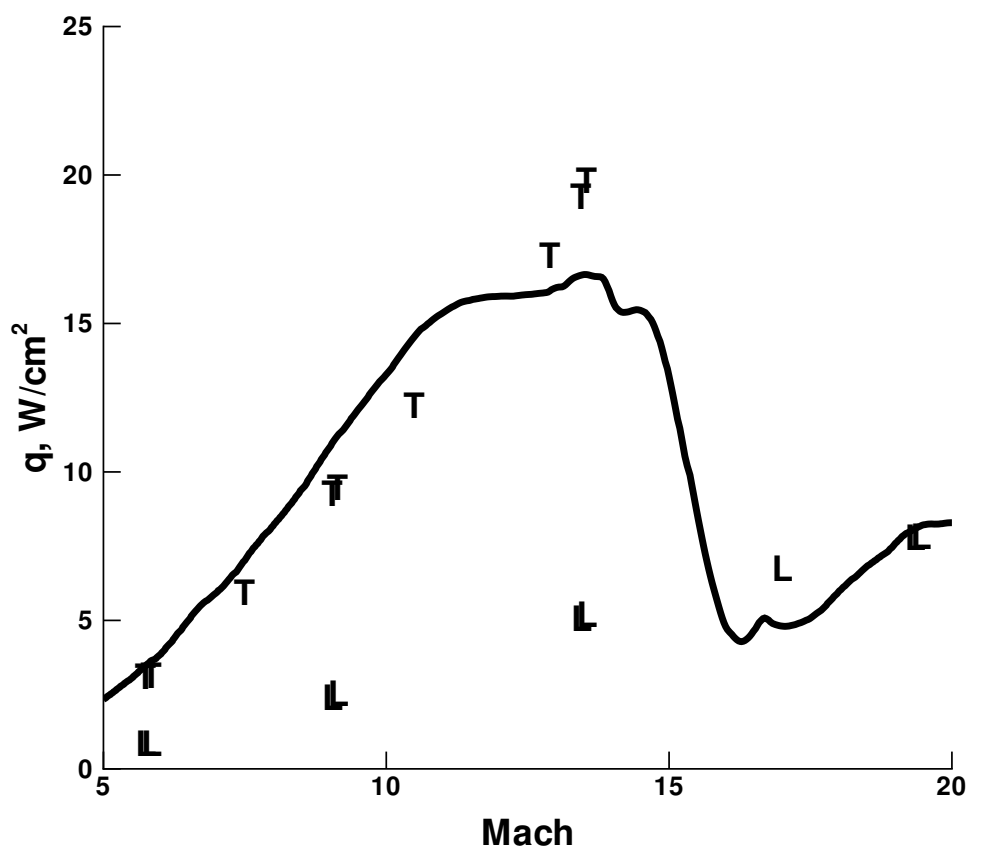

Figure 7. Convective heat transfer rates for TC-1, STS-119, compared to turbulent T and laminar L CFD simulations.

The numerical predictions show no such plateau in the Mach 11-15 heating rates as is observed in the flight data, with the predictions increasing monotonically with increasing Mach number. The true state of the flight boundary layer is difficult to interpret between Mach 11-15, and this difference in trends between prediction and measurement could be an inadequacy in the turbulence models or could mean that the real boundary layer flow was not in a fully-developed turbulent state. Another possibility is that the trip produces a trailing vortex interaction that persists to some extent beyond transition onset. Visual inspection of the orbiter tiles after landing show a trailing streak of high sheen, extending from the boundary layer experiment trip towards the TC- 1 vicinity, that is interpreted as being caused by a strong vortex interaction.

For Mach numbers less than 11, the numerical predictions underestimate the measurements, with improving agreement as the Mach number decreases. The turbulent computations match the measurement at Mach 6.

Convective heating rates from TC-2 during the STS-119 reentry are shown in figure 8. TC-2 is located about midway between the boundary layer trip and TC-1. The overall trends are similar to the TC-1 response. Oscillations in the heating history during the laminar and transitional period between Mach 12-20 suggest a strong interaction on the flow from a vortex, or similar disturbance, emanating from the trip. The measurements show something of a plateau between Mach 11-14, but there is a variation of about $2 \mathrm{~W} / \mathrm{cm}^{2}$ amplitude that indicates against the flow being of a fully-developed turbulent nature. As with the TC-1 comparison, the turbulent numerical predictions show no indication of a plateau trend. The measurements follow an expected turbulent response over the trajectory for Mach numbers below 11. The turbulent CFD results under-predict the measured heating rates for Mach numbers less than 11, with improving accuracy for lower Mach numbers. The turbulent simulation matches the flight data at Mach 6 .

Figure 9 shows the STS-119 convective heating rates at TC-90, located toward the aft end of the vehicle centerline. This area of the vehicle is not affected by the wake of the boundary layer trip, but rather was washed by turbulent flow emanating from an unidentified source near the nose of the vehicle, as observed in the STS-119 HYTHIRM imagery. ${ }^{3}$ The heating rates show a classic transition from laminar to turbulent heating, with transition onset at Mach 11 . The heating rates during transitional flow slightly over-shoot the fully-turbulent rates, at Mach 9. There is no plateau in the measured heating during the transitional phase. The Mach numbers below 8 follow the typical turbulent heating trajectory profile down toward landing. The turbulent computations match the measurements for Mach numbers less than 10.

Figure 10 shows that the TC-2 heating generally followed a similar trend on STS-128 as for STS- 119. 


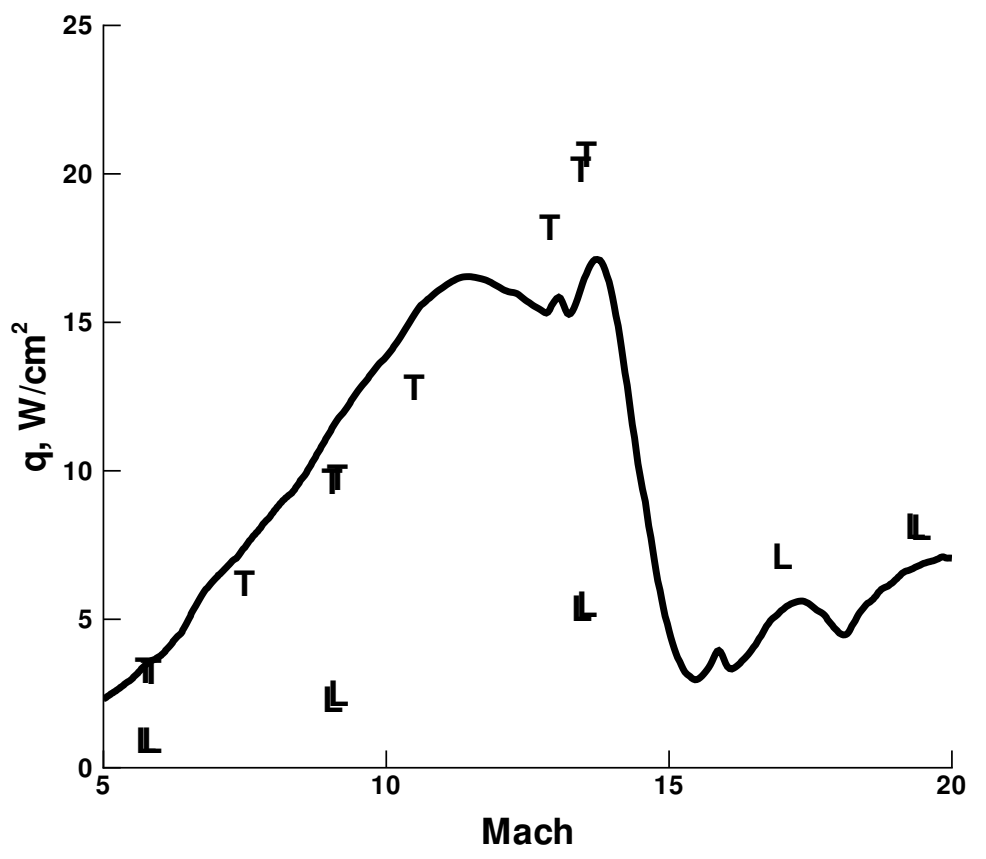

Figure 8. Convective heat transfer rates for TC-2, STS-119, compared to turbulent T and laminar L CFD simulations.

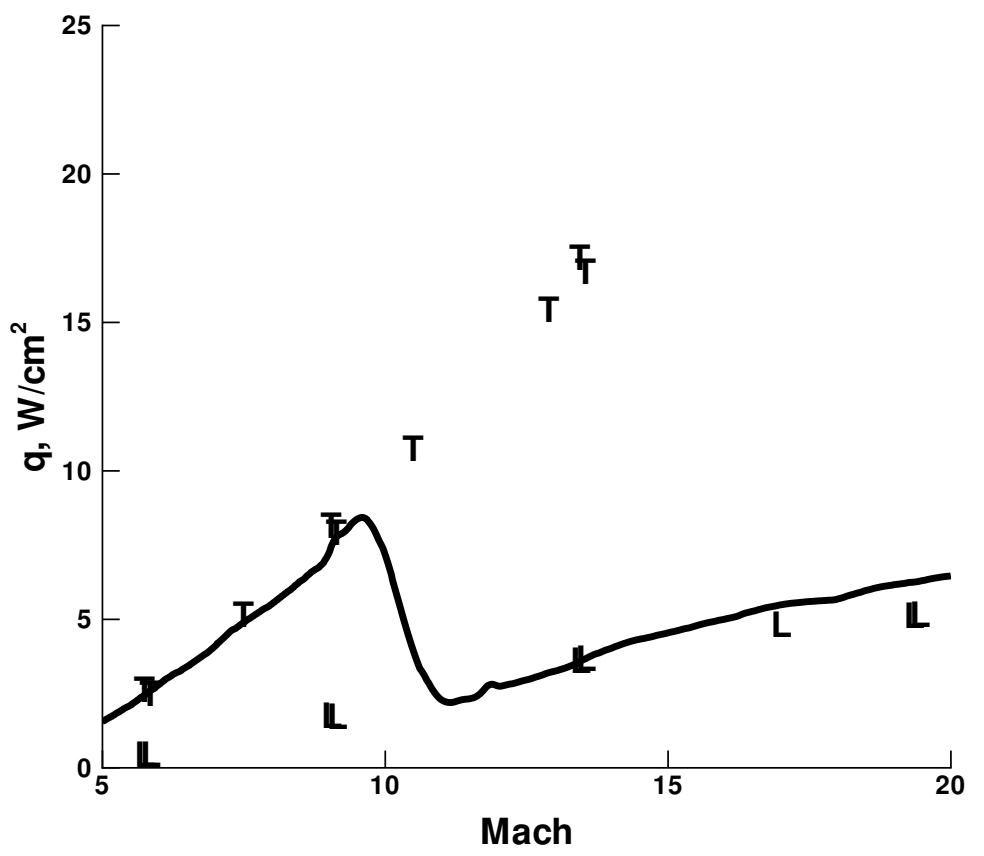

Figure 9. Convective heat transfer rates for TC-90, STS-119, compared to turbulent T and laminar L CFD simulations. 


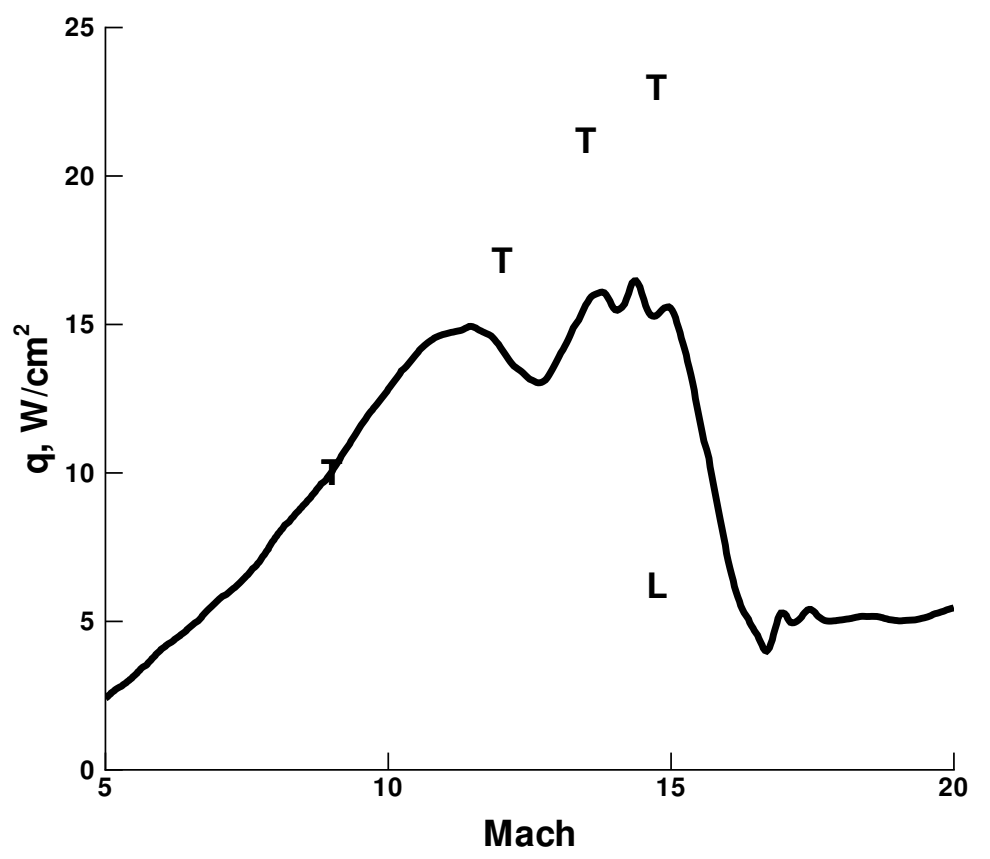

Figure 10. Convective heat transfer rates for TC-2, STS-128, compared to turbulent T and laminar L CFD simulations.

Transition begins at about Mach 16, earlier than on STS-119 because of the larger boundary layer trip on STS-128. Again, the post-transition flow in the Mach 11-15 range shows oscillations that may be driven by large-scale disturbances emanating from the trip. Peak measured heating rates are similar between STS-128 and STS-119, at about $16 \mathrm{~W} / \mathrm{cm}^{2}$. The turbulent numerical models again do a poor job of representing the physical flow between Mach 11-15. The numerical prediction at Mach 9 matches the flight measurement.

TC-6, located aft of the boundary layer trip and inboard of TC-1, exhibits a heating pattern intermediate between TC-1/2 and TC-90. figure 11 shows the STS-128 heating history for TC-6. The transition at TC-6 is delayed relative to TC-1, reflecting the temporal growth in lateral extent of the turbulent wedge emanating from the trip. Transition onset occurs at Mach 15, and the heating rises to a distinct peak of $14 \mathrm{~W} / \mathrm{cm}^{2}$. But this heating peak is considerably broader than is seen on the fuselage at TC-90. The numerical prediction at Mach 9 matches the flight measurement.

TC-80 and TC-97 are located symmetrically about the orbiter centerline, forward of the main landing gear doors at about mid-fuselage, and also forward of the flight experiment boundary layer trip. Heating rates from both thermocouples during the STS-128 flight are plotted in figure 12. Fuselage boundary layer transition was asymmetric, with TC-80 transitioning earlier than TC-97, at Mach 14 and 9, respectively. The CFD simulations enforced a symmetry plane, so the same predictions apply to both thermocouple locations. At this fuselage location, the transition from laminar to turbulent heating is more sharply defined than for any of the thermocouples in the wake of the boundary layer transition experiment trip, which was located on the wing. As with the other STS-128 comparisons, the turbulent Mach-9 predictions matches the turbulent flight data.

On STS-128, TC-90 experienced boundary layer transition at Mach 14, figure 13. The transition from laminar to turbulent heating on the fuselage centerline is abrupt, with a sharp peak in heating up to $13 \mathrm{~W} / \mathrm{cm}^{2}$. The turbulent CFD prediction at Mach 13.5, $13.8 \mathrm{~W} / \mathrm{cm}^{2}$, over-predicts the measurement, $12.4 \mathrm{~W} / \mathrm{cm}^{2}$. At Mach 9, the over-prediction is $8.2 \mathrm{~W} / \mathrm{cm}^{2}$ versus $7.7 \mathrm{~W} / \mathrm{cm}^{2}$.

In relation to the assessments of turbulent CFD modeling against the thermocouple temperatures, which have been reported in references 8 and 9 , the present casting in terms of heat transfer clarifies the numericalto-measured comparisons, but does not resolve the discrepancy in trends for Mach numbers greater than 11. There remains a difference between the CFD simulation and the physical flow, which is manifested as an observed plateau in heat transfer rate measured by the thermocouples downstream of the boundary layer transition flight experiment trip. This difference is not explained by thermal conduction effects into the tiles. 


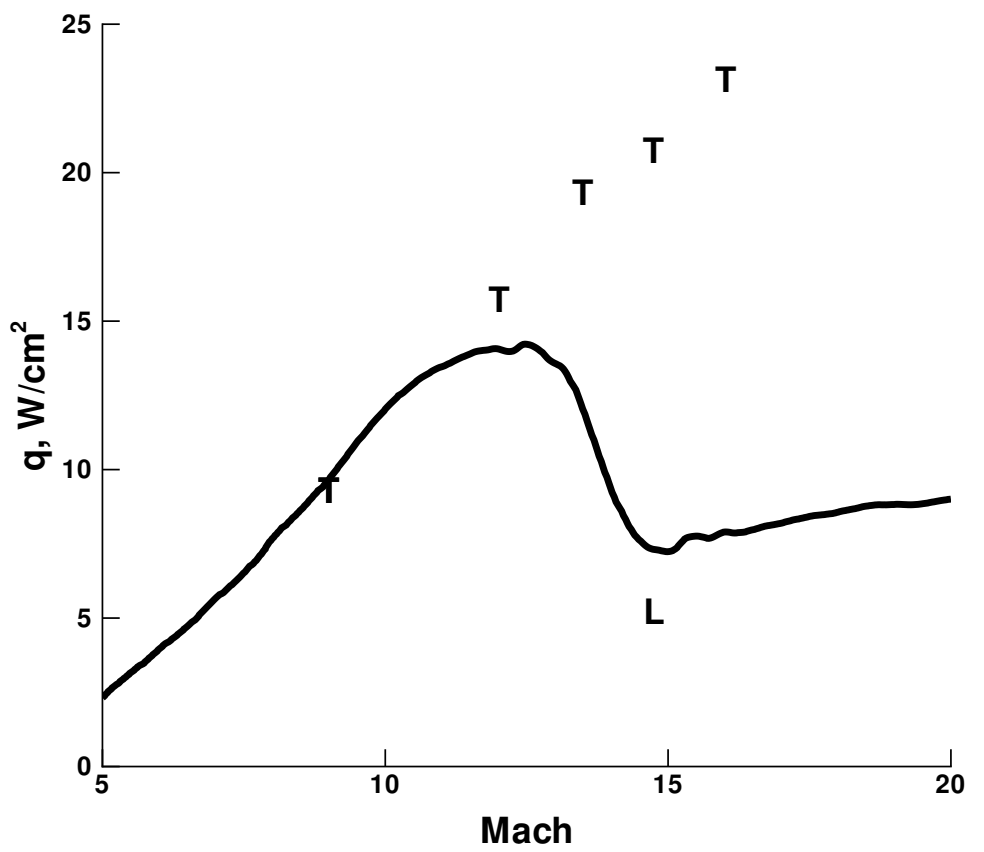

Figure 11. Convective heat transfer rates for TC-6, STS-128, compared to turbulent $T$ and laminar L CFD simulations.

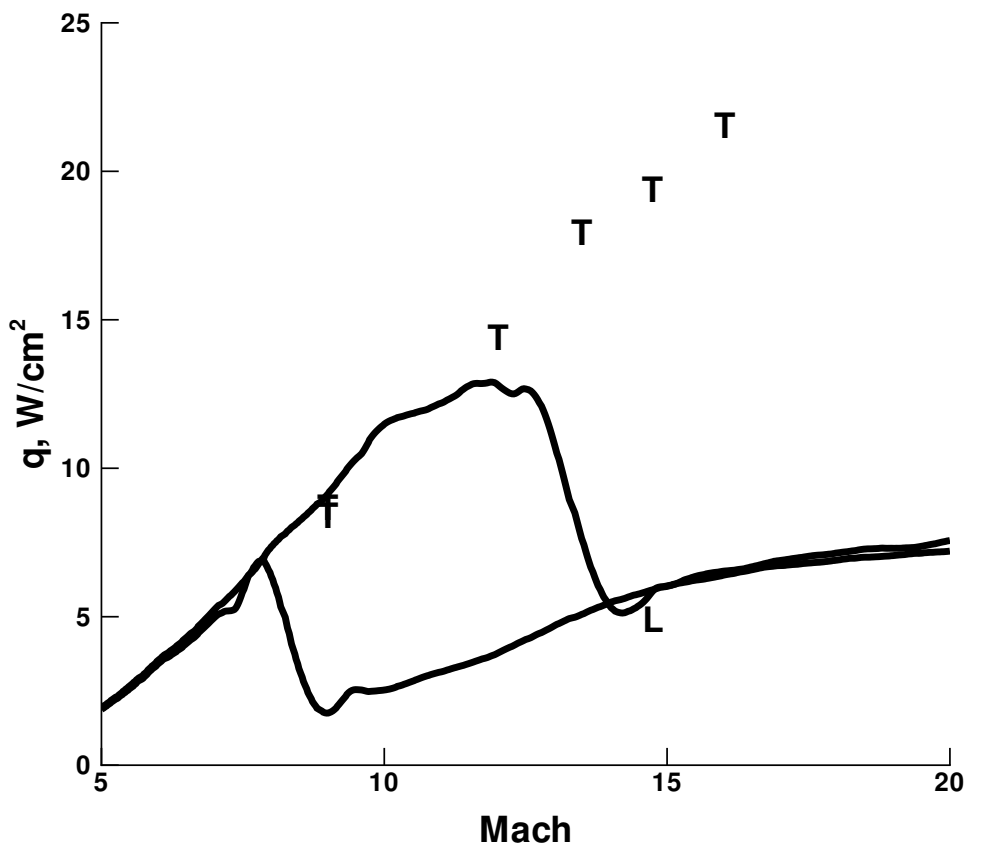

Figure 12. Convective heat transfer rates for TC-80 and TC-97, STS-128. compared to turbulent T and laminar L CFD simulations. TC-80, located to port, experienced higher Mach number transition than TC-97, located to starboard. 


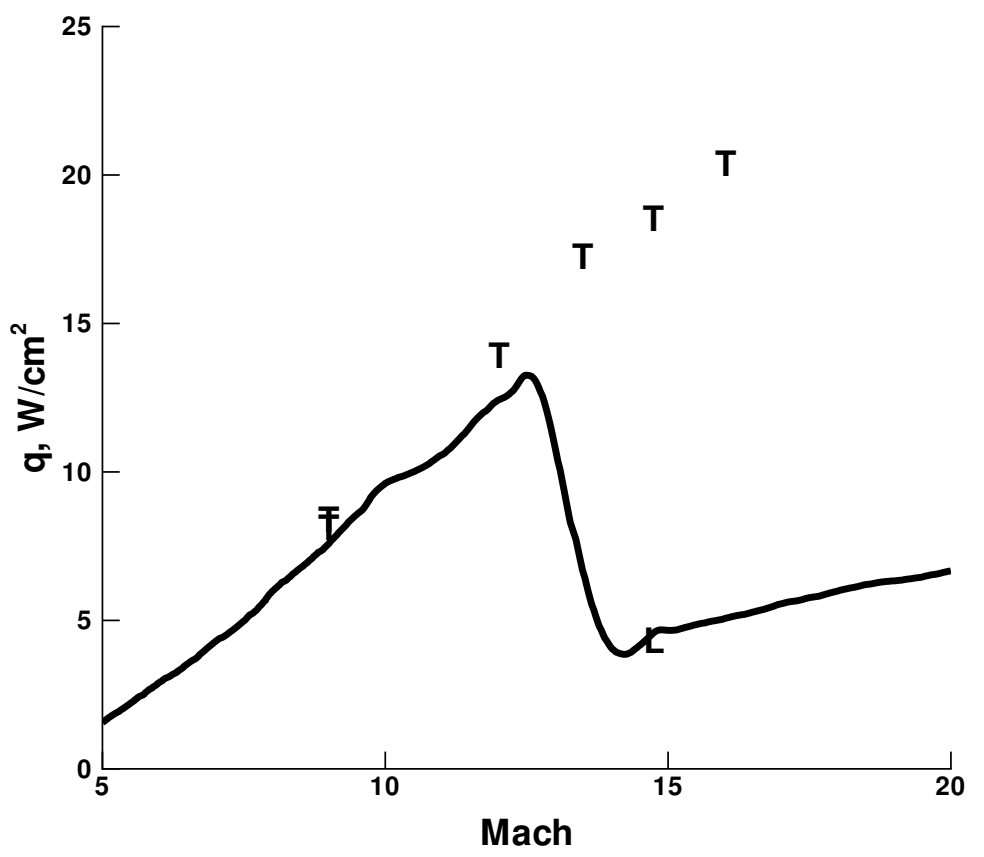

Figure 13. Convective heat transfer rates for TC-90, STS-128, compared to turbulent T and laminar L CFD simulations.

Some candidate causes for the diverging trend between turbulent CFD modeling and non-laminar flight data can be hypothesized. The CFD simulations do not include a transition length. The entire vehicle is wetted by turbulent flow, as opposed to having a wedge of turbulent flow within an otherwise laminar flow field. The boundary layer trip is included in the geometry model, and the grid is insufficient to resolve any trailing vortex that might emanate from the trip even if the trip were included in the geometry model. There may be an element in the nature of hypersonic turbulent flow that is missing from the turbulence models, such as a non-continuum, compressibility, or chemistry related effect.

\section{Summary of results}

The boundary layer transition experiments flown on the Space Shuttle orbiter Discovery during STS-119 and STS-128 provide rare hypersonic turbulent flow measurements. Prior attempts to validate turbulent CFD methods against the flight temperature measurements in the Mach 11-15 range have been equivocal, because of uncertainty in distinguishing fully-turbulent from transitional flow; the CFD models are for turbulent, not transitional, boundary layers. The present work transforms the surface temperature measurements into convective heat transfers, by means of a thermal conduction analysis. Using convective heat transfer as the figure of merit, turbulent CFD prediction methods are again assessed against the flight data, resulting in a clearer interpretation of the relation between turbulent flow models and the real physical flow produced by the flight experiment. Nonetheless, transient thermal conduction into the vehicle tiles does not resolve the discrepancy between modeling and reality for the Mach 11-15 flow in the wake of the boundary layer transition flight experiment trip.

\section{References}

\footnotetext{
${ }^{1}$ Anderson, B. P., Campbell, C. H., Saucedo, L. A., Kinder, G. R., and Berger, K. T., "Boundary Layer Transition Flight Experiment Overview and In-Situ Measurements," AIAA Paper 2010-240, Jan. 2010.

${ }^{2}$ Berger, K., Anderson, B., Campbell, C., Garske, Saucedo, and Kinder, G., "Boundary Layer Transition Flight Experiment Overview," AIAA Paper, June 2011, To be presented at 42nd AIAA Thermophysics Conference.

${ }^{3}$ Horvath, T. J., Tomek, D. M., Berger, K. T., Zalameda, J. N., Splinter, S. C., and Krasa, P. W., "The HYTHIRM Project: Flight Thermography of the Space Shuttle during Hypersonic Re-entry," AIAA Paper 2010-241, Jan. 2010.

${ }^{4}$ Wadhams, T. P., Holden, M. S., MacLean, M. G., Campbell, C., Anderson, B., Berger, K., Rufer, S., and Berry, S.,
} 
"Experimental Studies of Space Shuttle Orbiter Boundary Layer Transition with Flight Representative Protuberances," AIAA Paper, June 2011, To be presented at the 42nd AIAA Thermophysics Conference.

${ }^{5}$ Horvath, T. J., Kerns, R., Jones, K., Ruemmele, Schwartz, R., Gibson, and Dantowitz, "A Vision of Quantitative Imaging Technology for the Assessment of Advanced Flight Technologies," AIAA Paper, June 2011, To be presented at the 42nd AIAA Thermophysics Conference.

${ }^{6}$ Taylor, J., Gibson, D., Spisz, Horvath, T., Schwartz, R., Splinter, Zalameda, J., Kerns, R., and Tietjen, A., "Global Thermography of the Space Shuttle during Hypersonic Re-entry," AIAA Paper, June 2011, To be presented at the 42nd AIAA Thermophysics Conference.

${ }^{7}$ Schwartz, R., Verstynen, Gruber, Splinter, Horvath, T., and McCrea, "Remote Infrared Imaging of the Space Shuttle During Hypersonic Flight: HYTHIRM Mission Operations and Coordination," AIAA Paper, June 2011, To be presented at the 42nd AIAA Thermophysics Conference.

${ }^{8}$ Wood, W. A., Kleb, W. L., Tang, C. Y., Palmer, G. E., Hyatt, A. J., Wise, A. J., and McCloud, P. L., "Comparison of CFD Predictions with Shuttle Global Flight Thermal Imagery and Discrete Surface Measurements," AIAA Paper 2010-454, Jan. 2010.

${ }^{9}$ Wood, W. A., Kleb, W. L., and Hyatt, A. J., "Assessment of Turbulent CFD against STS-128 Hypersonic Flight Data," AIAA Paper 2010-4889, June 2010.

${ }^{10}$ Wright, M. J., Candler, G. V., and Bose, D., "Data-Parallel Line Relaxation Method for the Navier-Stokes Equations," AIAA Journal, Vol. 36, No. 9, Sept. 1998, pp. 1603-1609.

${ }^{11}$ Gnoffo, P. A., Gupta, R. N., and Shinn, J. L., "Conservation Equations and Physical Models for Hypersonic Air Flows in Thermal and Chemical Nonequilibrium," NASA TP 2867, Feb. 1989.

${ }^{12}$ Gnoffo, P. A., "An Upwind-Biased, Point-Implicit Relaxation Algorithm for Viscous, Compressible Perfect-Gas Flows," NASA TP 2953, Feb. 1990.

${ }^{13}$ Navier, M., "Mémoire sur les lois du Mouvement des Fluides," Mémoire de l'Académie des Sciences, Vol. 6, 1827, pp. 389.

${ }^{14}$ Stokes, G. G., "On the Theories of the Internal Friction of Fluids in Motion," Trans. Cambridge Philosophical Society, Vol. 8, 1849, pp. 227-319.

${ }^{15}$ Cebeci, T. and Smith, A. M. O., "A Finite-Difference Method for Calculating Compressible Laminar and Turbulent Boundary Layers," Journal of Basic Engineering, Sept. 1970, pp. 523-535.

${ }^{16}$ Baldwin, B. S. and Lomax, H., "Thin Layer Approximation and Algebraic Model for Separated Turbulent Flows," AIAA Paper 78-257, January 1978.

${ }^{17}$ External Aerothermal Analysis Team, "Smooth Outer Mold Line Aerothermal Solution Database for Orbiter Windside Acreage Environments During Nominal Entry Conditions," Engineering Note EG-SS-06-1, NASA Johnson Space Center, Houston, Texas, April 2005, Presented to Orbiter Configuration Control Board.

${ }^{18}$ Reuther, J., Thompson, R., Pulsonetti, M., and Campbell, C., "Computational Aerothermodynamic Analysis for the STS-107 Accident Investigation," AIAA Paper 2004-1384, Jan. 2004.

${ }^{19}$ Tang, C. Y., Trumble, K. A., Campbell, C. H., Lessard, V. R., and Wood, W. A., "Numerical Simulations of the Boundary Layer Transition Flight Experiment," AIAA Paper 2010-453, Jan. 2010.

${ }^{20}$ Amar, A., Calvert, N., and Kirk, B., "Development and Verification of the Charring Ablating Thermal Protection Implicit System Solver," AIAA Paper 2011-144, Jan. 2011.

${ }^{21}$ Beck, J., Blackwell, B., and St.C̃lair, C., Inverse Heat Conduction: Ill-posed Problems, Wiley-Interscience, New York, 1985.

${ }^{22}$ Savitzky, A. and Golay, J. E., "Smoothing and Differentiation of Data by Simplified Least Squares Procedures," Analytical Chemistry, Vol. 36, No. 8, July 1964, pp. 1627-1639.

${ }^{23}$ Madden, H., "Comments on the Savitzky-Golay Convolution Method for Least-Squares Fit Smoothing and Differentiation of Digital Data," Analytical Chemistry, Vol. 50, No. 9, Aug. 1978, pp. 1383-1386.

${ }^{24}$ Throckmorton, D. A., "Benchmark Determination of Shuttle Orbiter Entry Aerodynamic Heat-Transfer Data," Journal of Spacecraft and Rockets, Vol. 20, No. 3, May 1983, pp. 219-224. 